\title{
Pharmacological management of cachexia in adult cancer patients: a systematic review of clinical trials
}

\author{
Shailesh M. Advani ${ }^{1,3}$, Pragati G. Advani ${ }^{2}$, Helena M. VonVille ${ }^{3}$ and Syed H. Jafri ${ }^{4 *}$ (D)
}

\begin{abstract}
Background: Cachexia is a multisystem syndrome characterized by weight loss, anorexia, loss of muscle mass, systemic inflammation, insulin resistance, and functional decline. Management of cachexia involves addressing multiple underlying biological mechanisms. Previous review on pharmacological management of cancer cachexia identified progestins and corticosteroids as effective agents for treatment of cachexia. However, to date no consensus exists on a single effective or standard treatment for management of cachexia. The aim of this systematic review is to determine the effectiveness of pharmacological treatments used to manage cachexia among adult cancer patients.

Methods: We performed literature searches of PubMed (NLM), Embase (Ovid), and Medline(Ovid) to identify clinical trials focused on pharmacological management of cancer cachexia among adult cancer patients from 2004 to 2018. Three reviewers screened a random selection of abstracts to measure for interrater reliability. After this step, each screener screened two-thirds of all abstracts and 177 studies were identified for full text review. The primary outcome was impact of pharmacological management on change in either weight or lean body mass in cancer patients.
\end{abstract}

Results: We identified 19 articles (representing 20 RCTs) that focused on pharmacological management of cancer cachexia. Agents showing promising results included Anamorelin and Enobosarm. Anamorelin at 50 or $100 \mathrm{mg}$ per day for 12 weeks showed a consistent benefit across all studies and resulted in significant improvement in weight as compared to baseline among cancer patients. Enobosarm at 1 and $3 \mathrm{mg}$ per day was also effective in improving lean body mass and QOL symptoms among advancer stage cancer patients. Finally, use of combination agents provide evidence for targeting multiple pathways underlying cachexia mechanism to achieve maximum benefit. No agents showed functional improvement in cancer patients.

Conclusion: Anamorelin as a single agent shows promising results in improving cachexia related weight loss among cancer patients. Further research on combination therapies may be helpful to address critical gaps in cachexia management.

Keywords: Cancer cachexia, Weight loss, Sarcopenia, Systemic inflammation, Anamorelin

\section{Background}

Cancer cachexia remains a significant challenge in the management of cancer patients as no clear standard of care exists. Cancer Cachexia impacts approximately $60 \%$ of the 1.4 million patients diagnosed with cancer in the United States each year $[1,2]$. Among late stage patients, cachexia affects $50-80 \%$ patients and is responsible for

\footnotetext{
* Correspondence: Syed.H.Jafri@uth.tmc.edu

${ }^{4}$ Department of Medicine, Division of Oncology, The University of Texas Health Science Center at Houston, McGovern Medical School, Houston, TX 77030, USA

Full list of author information is available at the end of the article
}

$20 \%$ of cancer related deaths [3]. Prevalence of cachexia varies across cancer types and ranges from $60 \%$ among lung cancer patients, to about $80 \%$ among gastrointestinal cancer (pancreas, stomach, colorectal and esophagus) patients [4]. Clinically, cachexia is defined through a consensus definition of weight loss of $\geq 5 \%$ of body weight in the past 6 months or $\geq 2 \%$ loss in patients with body mass index (BMI) of $<20 \mathrm{~kg} / \mathrm{m}^{2}$ [5]. In addition to weight loss, patients with cancer cachexia suffer from multisystem syndrome characterized by anorexia, loss of muscle mass, systemic inflammation, insulin resistance,

(c) The Author(s). 2018 Open Access This article is distributed under the terms of the Creative Commons Attribution 4.0 International License (http://creativecommons.org/licenses/by/4.0/), which permits unrestricted use, distribution, and reproduction in any medium, provided you give appropriate credit to the original author(s) and the source, provide a link to the Creative Commons license, and indicate if changes were made. The Creative Commons Public Domain Dedication waiver (http://creativecommons.org/publicdomain/zero/1.0/) applies to the data made available in this article, unless otherwise stated. 
and functional decline [2, 6, 7]. Furthermore cachexia patients have also shown to have anemia, hypoalbuminemia, and asthenia [8]. In addition, cachexia has been studied to influence clinical outcomes by influencing response to chemotherapy in cancer patients $[9,10]$.

The exact etiology of cancer cachexia remains unknown. Patients with certain types of cancers (lung, pancreas, esophagus and head and neck) are more likely to experience weight loss/cachexia than patients with cancers of the breast and sarcomas [11]. The prognostic effect of cachexia was demonstrated by DeWys et al. (1980) when they reviewed 3047 cancer patients, in which patient-reported weight loss of $\geq 6 \%$ in the preceding 6 months was associated with poor outcomes amongst all cancer types [12]. Inflammation is thought to be a prominent mechanism underlying cachexia, including increase levels of inflammatory cytokines like Interleukin (IL)-6, tumor necrosis factor (TNF) $-\alpha$ and IL-1 [13-15]. Muscle wasting is one of the hallmarks of cancer cachexia which involves proteolysis mediated by the ubiquitin-proteasome pathway (UPP) through cytokine activated signaling molecules like NF-kB, the p38MAPK or JAK-STAT3 pathway and the autophagy-lysosome pathway (ALP) [16, 17] Toll-Like receptor 4 (TLR4), a transmembrane receptor expressed on immune cells and skeletal muscles have also been shown to act as central mediator in cachexia pathophysiology, both for cancer-induced muscle protein breakdown and increased release of cytokines [18].

Management of cancer cachexia remains challenging, due to multiple reasons involving differences in predisposition of cancer types, underlying multiple pathophysiological processes and concurrent disease process among cancer patients. Despite several randomized clinical trials involving a variety of agents, no single gold standard or Food and Drug Administration (FDA) approved agent exists for cachexia management. A systemic review published in 2005, identified strong evidence in favor of progestins such megesterol acetate (MA) and short course of corticosteroids as appetite stimulants in cancer patients [19]. However, MA was linked with a higher chance of developing deep venous thrombosis, and the benefit of corticosteroids was short-lived [6, 7]. Since 2004, cancer cachexia has been defined through a standardized consensus definition, and several agents with different mechanism of actions have been studied [5]. The aim of this systematic review was to provide an update on pharmacological agents used for cachexia management, determine the effectiveness of these agents and provide a summary of their impact on clinical measures of cachexia in adult cancer patients. The primary outcomes of interest were changes in weight and/or change in lean body mass. Secondary outcomes were changes in appetite, physical functioning and quality of life indicators. Finally, we assessed for impact of these pharmacological agents on overall survival.

\section{Methods \\ Search methodology}

This systematic review was registered with PROSPERO (registration number CRD42016042422). Eligibility criteria were developed a priori and required that studies had to be focused on the pharmacological management of cancer-related cachexia among adults and had to include a clear definition of defining cachexia among cancer patients. We excluded studies examining cachexia among non-cancer populations including those with chronic comorbid conditions or infectious diseases including HIV. The primary outcome of study was change in weight and/or lean body mass (Outcome 1). Secondary outcomes included impact of pharmacological agents on measures of appetite (Outcome 2), physical functioning (e.g. grip strength) (Outcome 3), quality of life (Outcome 4), and overall survival (Outcome 5). We included original peer-reviewed articles that included data from randomized controlled trials (RCTs) on pharmacological agents used to treat cachexia among cancer patients, were published in English language from 2004 to 2018, and excluded all comments, editorials, conference abstracts, and prior reviews.

We searched Medline (Ovid), PubMed (NLM), and Embase (Ovid) with the help of a health sciences librarian (H.V.) with systematic review experience who developed all searches. The initial searches were completed in April 2016; an updated PubMed search was completed July 2018. A combination of MeSH terms and title, abstract, and keywords was used to develop the initial Medline search. The search was then adapted to search other databases. Additional file 1 provides the search strategies used for each database. RefWorks (ProQuest) was used to store all citations found in the search and to check for duplicates. Search strategies and results were tracked using one of a series of Excel workbooks designed specifically for systematic reviews [20].

An online random number generator (https:// www.random.org/integers) was used to create a random sample of 166 numbers that were then input into an Excel workbook designed specifically for the interrater reliability test [20]. These numbers corresponded to line numbers within the Excel workbook, resulting in a random sample of titles and abstracts; authors and journal titles were not included in the sample. Three authors (S.A., P.A., and S.J.) independently screened $166 \mathrm{ab-}$ stracts and reached weak agreement (S.A. and P.A., Cohen's $\mathrm{K}=0.50$; P.A. and S.J., Cohen's $\mathrm{K}=0.34$; S.A. and S.J. Cohen's $K=0.40)$. Due to weak agreement, the reviewers mutually discussed their disagreements and 
received additional training by S.J. on the scope of projects including an overview of pharmacological agents, definitions of key outcomes and examples of abstracts that fit the inclusion criteria. Following additional training and still blinded to authors and journal titles, each reviewer independently screened two-thirds of the titles and abstracts with each item being screened by two authors. All data were then combined into a single screening workbook, and major disagreements after full review were further discussed to meet consensus. All studied considered for inclusion were independently reviewed by two authors (S.A. and S.J.) and consensus was again reached by discussion to any disagreements at this step. A list of excluded citations can be requested from the correspondence author (S.J.).

Data extraction and study quality: An excel workbook was developed to abstract relevant data from included studies. The key data abstracted include: pharmacological agents used; use of a control or comparison arm; study participant characteristics; dose and duration of management strategy; outcome measures (primary and secondary); and adverse events associated with primary pharmacological agent.

Quality Assessment: Quality of included studies was based on GRADE criteria. Evidence of bias was classified as low, moderate or high based on a list of criteria mentioned in GRADE handbook [21].

\section{Results}

Our search identified 6589 abstracts in the initial screening criteria. After removal of duplicates, three authors reviewed $2 / 3$ of abstracts and identified 19 publications to be included in the final review. (Fig. 1). Hence the final review included 19 articles, reporting on 20 clinical trials that focused on clinical trials on pharmacological management of cachexia (identified through self-report or clinical definition) among cancer patients. Key terms included cachexia, anorexia, or cancer cachexia-anorexia syndrome (CACS). Table 1 summarizes our study characteristics and outcomes.

\section{Study/patient characteristics}

Fourteen studies reported on comparing single agents, and five studies reported on combination agents for cachexia management. A placebo arm was present in 12 of the 19 included articles. All patients had inoperable or advanced cancer (stage III/IV). Nine studies were cancer specific and 10 had multiple cancer types. Cachexia was defined as weight loss ranging from $\geq 2 \%$ to $\geq 10 \%$ of body weight in the preceding months but most studies defined it as weight loss $\geq 5 \%$ of body weight $(n=13)$. Outcome measures varied across studies and included change in total body weight, lean body mass (LBM), functional improvement (e.g., handgrip strength [HGS]), changes in appetite, or improvement in QOL and overall survival. Few

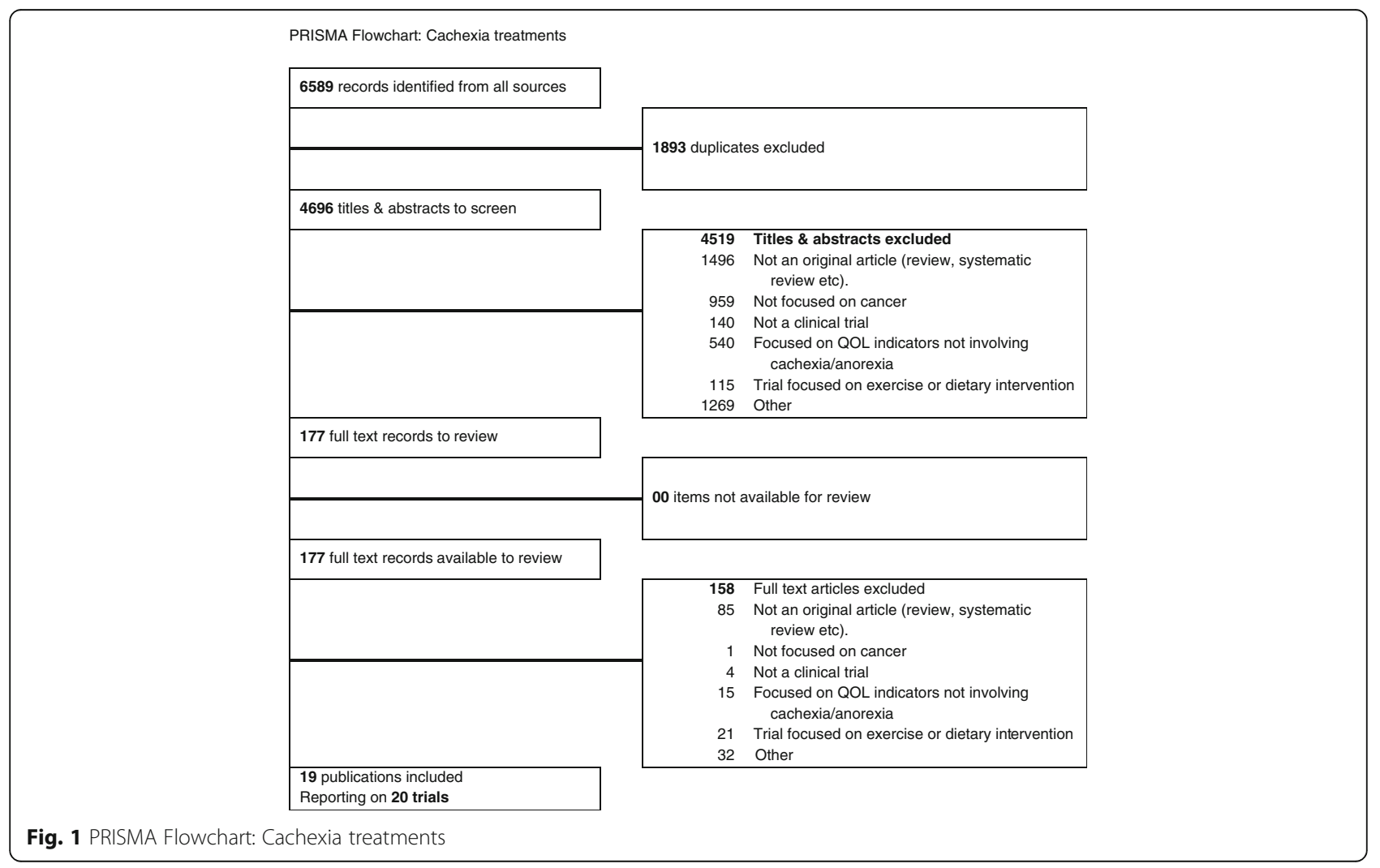




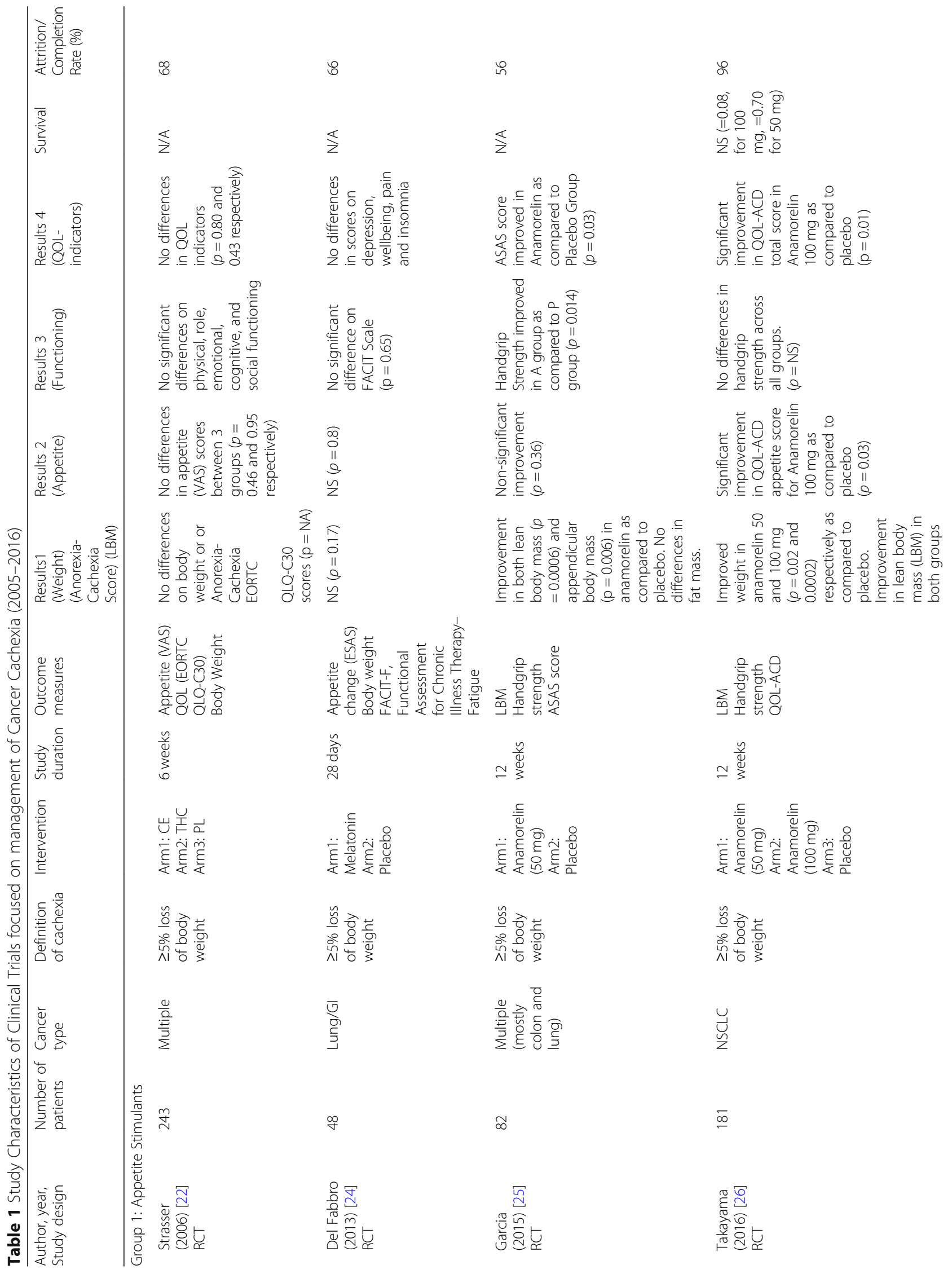




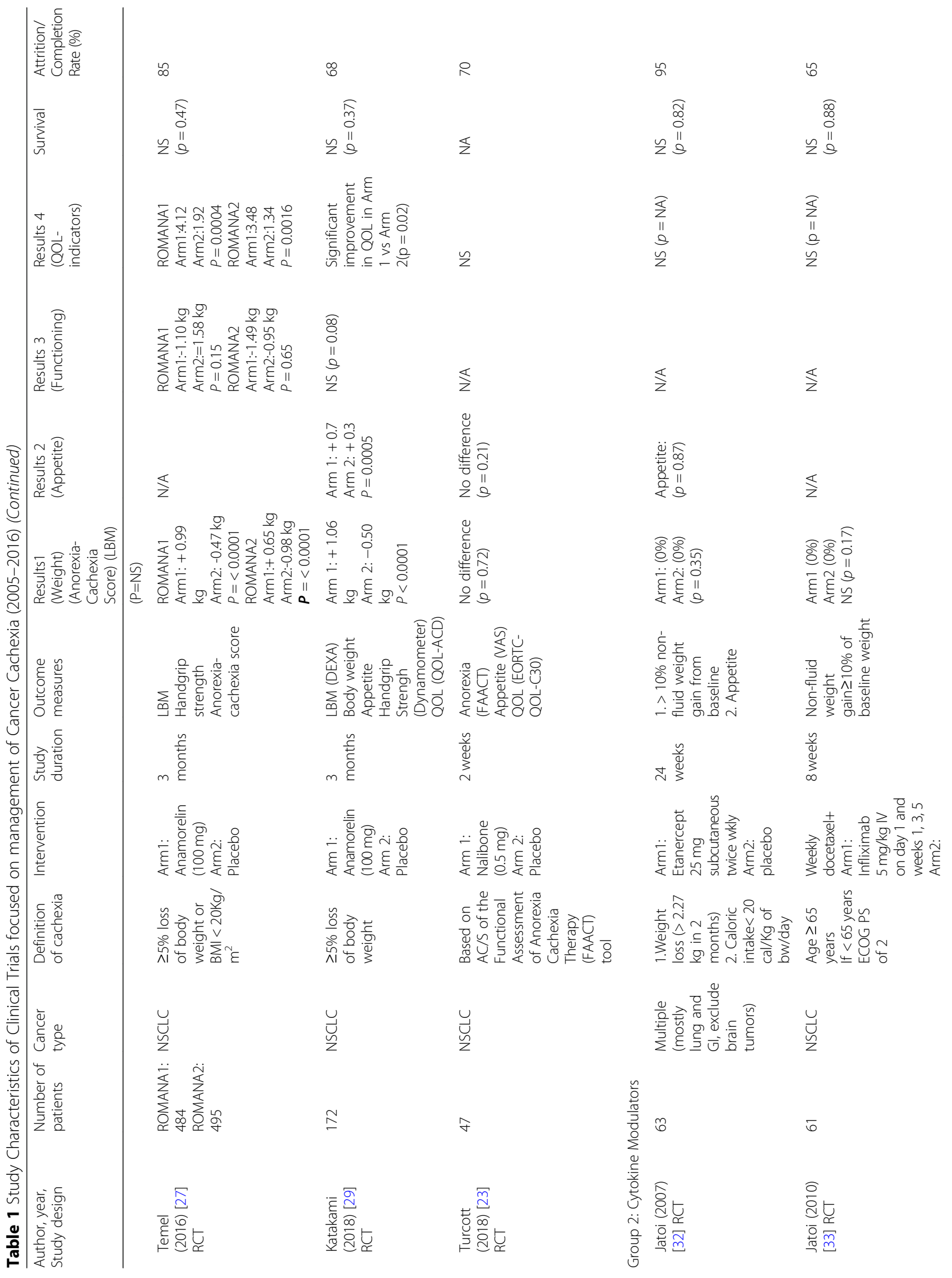




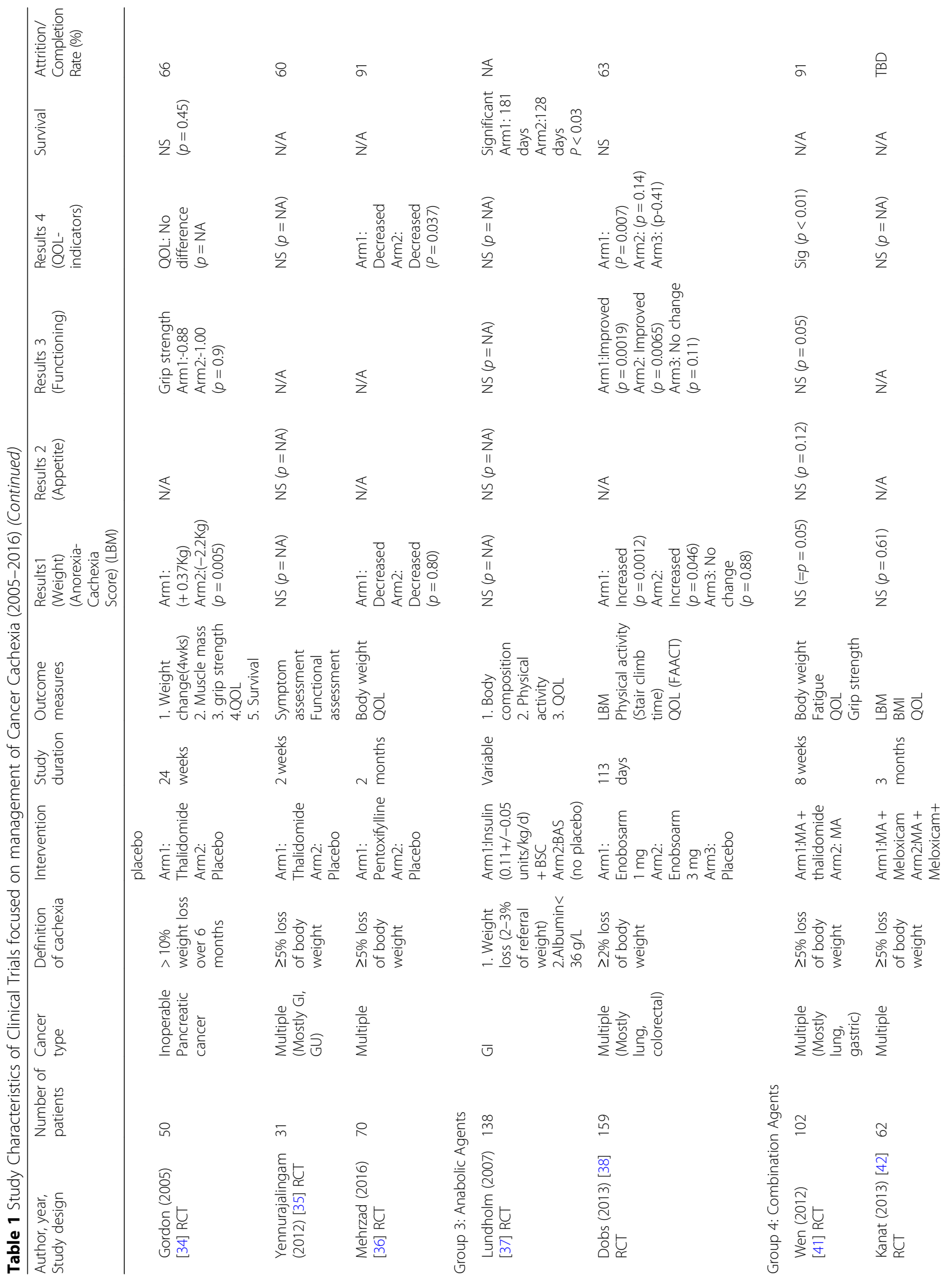




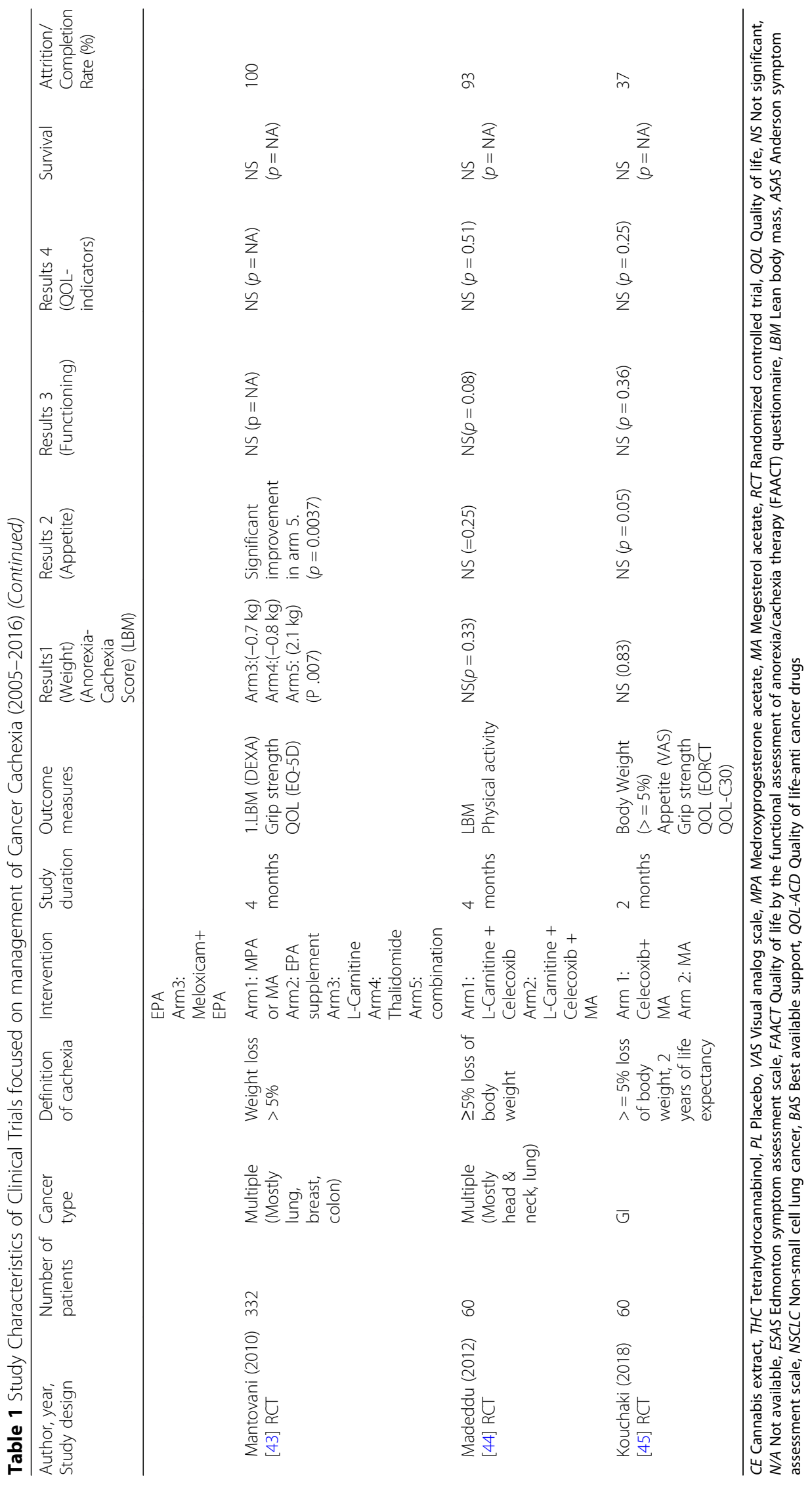


studies also assessed for changes in levels of cytokines including IL-6 and TNF-alpha (Additional file 2).

\section{Classification of pharmacological agents}

We tried to characterize pharmacological agents for cancer cachexia into 4 groups based on their mechanism of action. These include: (A) Appetite Stimulants, (B) Cytokine Modulators, (C) Anabolic agents and (D) Combination therapies.

\section{Appetite stimulants}

These include Anamorelin, Cannabis Sativa, Melatonin, and Nabilone.

\section{Cannabis sativa}

One study compared cannabis sativa and its derivatives including delta-9-tetrahydrocannabinol (THC) and cannabidiol (CBD) with placebo among patients with advanced cancer and $>5 \%$ weight loss in the previous 6 months [22]. Total of 243 Patients were randomized in a 2:2:1 ratio to receive cannabis extract (CE- standardized $2.5 \mathrm{mg}$ of THC and $1 \mathrm{mg}$ of CBD), THC (2.5 mg), or placebo for 6 weeks. Outcomes assessed included appetite improvement using the visual analog scale (VAS, $0 \mathrm{~mm}$ = worst, $100 \mathrm{~mm}=$ best), body weight change and QOL using the European organization for research and treatment of cancer QOL questionnaire C30 (EORTC QLQ-C30). No significant differences between groups on appetite, weight change or QOL scores were observed at the end of 6 weeks [22].

\section{Nabilone}

Similar to THC, derivates of Nabilone (synthetic analogue of $\Delta-9$ tetrahydrocannabinol (THC) has been approved for chemotherapy induced nausea and vomiting [23]. One study compared the effect of Nabilone vs placebo among patients with lung cancer and self-reported weight loss of $>5 \%$ in the past 6 months [23]. Total of 47 patients were randomized to either Nabilone $(0.5 \mathrm{mg} / 2$ weeks followed by $1.0 \mathrm{mg} / 6$ weeks) or placebo for period of 8 weeks. End of treatment evaluation reported no significant difference in weight, appetite or QOL between two groups [23].

\section{Melatonin}

One study compared the effect of melatonin to placebo on patients with advanced lung or GI cancers and cachexia (> 5\% loss of body weight) for 28 days [24]. The primary outcome was change in appetite using the Edmonton symptoms assessment scale (ESAS). The study was closed when 48 patients were recruited due to reasons of non-inferiority and interim analysis showed no difference between groups for endpoints on appetite or weight gain, as outlined by the Data Monitoring and
Safety board review in the study article [24]. In addition, no differences were observed for quality of life scores, fatigue levels or levels of C-reactive proteins and overall survival.

\section{Anamorelin}

Five studies compared the effect of anamorelin as compared to placebo on management of cancer cachexia [25-27]. Anamorelin is an orally available, selective ghrelin receptor agonist. Garcia et al. reported pooled analysis from two phase- 2 clinical trials in 44 patients with multiple cancers and cancer cachexia ( $>5 \%$ loss of body weight) [25]. Patients were randomized to anamorelin $50 \mathrm{mg}$ orally once daily or placebo. Outcomes included change in lean body mass (LBM) using dual-energy $\mathrm{x}$-ray absorptiometry, body weight and non-dominant grip strength as measured by dynamometer. Patients in the anamorelin group improved LBM by a mean of $1.89 \mathrm{~kg}$ (95\% confidence interval [CI], 0.84$2.95)$ as compared to a decrease of $-0.20 \mathrm{~kg}(95 \% \mathrm{CI}$, $1.23-0.8 ; P=0.0006$ ) in the placebo arm. There was no difference in handgrip strength between the two groups [25]. In addition, patients in anamorelin group also showed significant improvement in QOL scores and body weight, and no significant improvement in appetite scores. In addition, study participants also showed improvement in levels of IGF-1, IGFB-3, glucose and insulin.

Takayama et al (2016) reported a phase 2 RCT of anamorelin in 181 Japanese patients with advanced nonsmall cell lung cancer (NSCLC) and cachexia ( $\geq 5 \%$ loss of body weight). Patients were randomized to anamorelin $50 \mathrm{mg}, 100 \mathrm{mg}$, or placebo. The co-primary end points were changes in LBM and HGS. LBM improved by $0.55,0.85$, and $1.15 \mathrm{~kg}$ in the placebo, anamorelin 50 $\mathrm{mg}$, and $100 \mathrm{mg}$ groups, respectively $(P=0.05$, placebo vs. anamorelin $100 \mathrm{mg}$ ). There was no difference in HGS among the groups $(P=0.35)$ [26]. Patients in $100 \mathrm{mg}$ anamorelin group showed significant improvements in appetite scores, body weight, body fat, and QOL scores. No differences in levels of inflammatory markers including IL-1 and TNF-a were observed between groups. No significant differences in overall survival were observed between three groups.

The largest study of anamorelin was reported by Temel et al [27]. ROMANA 1 and ROMANA 2 were multinational randomized double-blind placebo controlled phase 3 clinical trials in patients with stage III and IV NSCLC with cancer cachexia ( $\geq 5 \%$ loss of body weight or body mass index $\left.<20 \mathrm{~kg} / \mathrm{m}^{2}\right)$. Patients were randomly assigned 2:1 to receive anamorelin $100 \mathrm{mg}$ or placebo for 12 weeks. Co-primary efficacy end points were change in LBM (measured via dual-energy X-ray absorptiometry) and HGS. A total of 484 patients were 
enrolled in ROMANA1 and 495 in ROMANA 2. Over 12 weeks, LBM improved in patients receiving anamorelin in ROMANA 1 (median increase $0.99 \mathrm{~kg}$ [95\% CI, 0.61-1.36] vs. placebo $-0.47 \mathrm{~kg}$ [95\% CI, $-1.00-0.21]$; $P \leq 0.0001)$. In ROMANA 2 the LBM increased by $0.65 \mathrm{~kg}$ (95\% CI, 0.38$0.91)$ compared with $-0.95 \mathrm{~kg}$ (95\% CI, $-1.49-10.41 ; P<$ $0.00001)$. There was no difference in HGS in ROMANA 1 $(-1.10 \mathrm{~kg}$ [95\% CI, -1.69 to -0.40$]$ vs. $-1.58 \mathrm{~kg}$ [95\% CI, -2.99 to -1.14$] ; P=0.15)$ or ROMANA $2(-1.49 \mathrm{~kg}[95 \%$ CI, - 2.06-0.58] vs. $-0.95 \mathrm{~kg}$ [95\% CI, - 1.56-0.04]; $P=$ 0.65 ) between anamorelin and placebo. There was no significant difference in survival between the treatment groups in either study [27]. Patients in ROMANO-1 and 2 were further invited into ROMANO-3 a safety extension study of anamorelin in NSCLC patients [28]. This study enrolled 513 patients from ROMANO I and II and reported sustained effect of anamorelin as compared to placebo on weight gain and appetite [28].

Finally, a recent study by Katakami et.al compared anamorelin to placebo among 174 Japanese patients diagnosed with stage III/IV NSCLC [29]. Patients were included who experienced $>5 \%$ loss of body weight and were randomized to either anamorelin $(100 \mathrm{mg})$ or placebo for 3 months. Primary end points were change in lean body mass (measured using DEXA) and body weight. Additional secondary end points included change in appetite scores (VAS), handgrip strength (measured using dynamometer) and overall QOL (measured using the QOL-ACD (Quality of Life-Anti Cancer Drugs) inventory). Patients in Anamorelin group reported a significant increase in LBM as compared to placebo (gain of $1.06 \mathrm{~kg}$ in anamorelin group vs loss of $0.5 \mathrm{~kg}$ in placebo group, $p$ $<0.001)$. Anamorelin was also associated with an overall improvement in appetite and QOL as compared to placebo group, however did not impact overall survival [29].

Across four studies on RCTs involving anamorelin, patients reported increased frequency of nausea, hyperglycemia, skin rash, first degree atrioventricular block and increased levels of c-glutamyltransferase [25-27, 29]. A recent meta-analysis on these studies confirmed the positive impact of anamorelin on lean body mass and quality of life among cancer patients, however it faced limitations including high heterogeneity observed due to limited sample size [30]. Additionally, Nishe et.al performed systematic review and meta-analysis on efficacy of anamorelin on weight gain and other outcomes among NSCLC patients and confirmed a significant positive effect of anamorelin on weight gain and QOL, and no effect on handgrip strength and overall survival in pooled analysis of 5 studies (6 RCTs) [31].

\section{Cytokine modulators}

These include Etanercept, Infliximab, Pentoxifylline and Thalidomide.
Etanercept Jatoi et.al enrolled 63 patients with advanced malignancy and cancer cachexia (weight loss of $\geq 2.27 \mathrm{~kg}$ over 2 months and/or daily intake of $<20 \mathrm{cal} / \mathrm{kg}$ body weight) and randomly assigned to receive etanercept at a dose of $25 \mathrm{mg}$ subcutaneously twice weekly versus placebo for 24 weeks [32]. The primary outcome was nonfluid weight gain of $10 \%$ compared with placebo. None of the patients in either arm achieved 10\% improvement in weight. Moreover, no improvement in appetite or QOL was observed in either arm. There was no difference in median survival between the groups (175 vs. 148 days in the etanercept vs. placebo groups, respectively; $P=0.82$ ) [32].

Infliximab In a double-blind RCT of patients with late stage non-small cell lung cancer (NSCLC), elderly patients ( $\geq 65$ years old) or $<65$ and with poor performance status (PS of 2$)(n=61)$ were randomized to receive infliximab $5 \mathrm{mg} / \mathrm{kg} /$ day IV on day 1 and weeks 1,3 , and 5 of the first 8-week cycle followed by day 1 on weeks 1 and 5 of every 8 -week cycle or placebo. Infliximab is a chimeric IgG1 kappa monoclonal antibody that blocks TNF- $\alpha$ by binding to it. All patients received docetaxel chemotherapy. The primary outcome was percentage of patients with nonfluid weight gain of $\geq 10 \%$ of baseline weight. None of the patients gained $\geq 10 \%$ of body weight compared with baseline, and the trial was closed early. There was no improvement in appetite. Patients receiving infliximab reported greater fatigue and treatment associated death with infliximab occurred in one patient. There was no significant difference in overall survival between two groups [33].

Thalidomide Two studies compared thalidomide to placebo for management of cachexia among cancer patients $[34,35]$. Thalidomide has immunomodulatory and anti-inflammatory properties and inhibits synthesis of TNF- $\alpha$. Fifty patients with inoperable pancreatic cancer and cancer cachexia ( $\geq 10 \%$ weight loss in the preceding 6 months) were randomized to receive thalidomide 200 mg orally daily or placebo for 24 weeks [35]. The primary outcome was change in body weight at 4 weeks. At the end of 4 weeks, patients in the thalidomide group had gained on average $0.37 \mathrm{~kg}$ in weight and $1.0 \mathrm{~cm}^{3}$ in arm muscle mass (AMA) compared with a loss of 2.21 kg weight and $4.46 \mathrm{~cm}^{3}$ AMA $(P=0.005$ and $P=0.002$, respectively) in the placebo group. There was no difference in HGS, QOL, or survival between the two groups [35]. In addition, treatment with thalidomide was associated with constipation, peripheral neuropathy, and rash.

In another study, 31 patients with advanced cancer of various types and cancer cachexia ( $\geq 5 \%$ weight loss in the preceding 6 months) currently not receiving chemotherapy were randomized to receive thalidomide $100 \mathrm{mg}$ 
orally daily versus placebo for 14 days. The primary outcome was symptom improvement using the ESAS and Functional Assessment of Chronic Illness Therapy Fatigue (FACIT-F). Twenty-one of 31 patients could complete the study. There was no significant difference in symptomatic improvement or levels of various cytokines between the two groups [34].

Pentoxifylline In an RCT, 70 Iranian patients with various advanced malignancies and cancer cachexia $(\geq 5 \%$ loss of body weight) were randomized to receive pentoxifylline $400 \mathrm{mg} 3$ times daily for 2 months or placebo [36]. Primary outcome was improvement in body weight. Pentoxifylline is a xanthine derivative that downregulates production of TNF- $\alpha$. Body weight and arm circumference decreased in both groups at 4 and 8 weeks with no significant difference between them. QOL as measured by SF-36 improved in the pentoxifylline group at 4 weeks $(P=0.029)$, but the effect did not last at 8 weeks $(P=$ $0.35)[36]$

\section{Anabolic agents}

Insulin Insulin was studied for its anti-lipolytic effects in 138 patients with advanced GI malignancies and cancer cachexia (weight loss 2-3\% of baseline and albumin $<36 \mathrm{~g} / \mathrm{L}$ ) [37]. Patients were randomized to receive long-acting insulin $0.11 \pm 0.05 \mathrm{U} / \mathrm{kg} /$ day subcutaneously once daily at an increasing dose to approach from 10 to $16 \mathrm{U} /$ day + best supportive care (BSC) (Indomethacin, erythropoietin treatment, and nutrition care) or BSC only without placebo. The BSC included oral indomethacin $25-50 \mathrm{mg}$ twice daily, recombinant erythropoietin 12,000-40,000 IU/week and specialized nutritional care based on pre-specified criteria. Outcome measures included body composition using dual energy X-ray absorptiometry, blood chemistries, indirect calorimetry for resting energy expenditure, maximum exercise test and QOL using SF-36 and EORTC QOL40). Insulin treatment for $193 \pm 139$ days significantly stimulated carbohydrate intake and increased whole-body fat without affecting fat-free lean tissue mass. There was no improvement in maximum exercise capacity or spontaneous physical activity. There was no improvement in appetite, body weight or QOL. In addition, significant differences in levels of serum fatty acids level were observed between two groups. There was improvement in survival in the insulin treated groups $224 \pm 163$ days compared with no treatment $175 \pm 148$ days $(P \leq 0.03)$ [37].

Enobosarm In a RCT, 159 patients with advanced cancer of different types and cachexia ( $\geq 2 \%$ weight loss in the preceding 6 months) were randomized to receive enobosarm $1 \mathrm{mg}$, enobosarm $3 \mathrm{mg}$, or placebo for up to 113 days [38]. Enobosarm, also termed as GTx-024 is an oral nonsteroidal selective androgen receptor modulator (SARM) [39]. Enobosarm has been shown to have tissue-selective anabolic and androgenic activity, and can increase muscle mass and function $[38,40]$. The primary endpoint was change in LBM from baseline assessed by dual-energy X-ray absorptiometry. Both enobosarm arms, 1 and $3 \mathrm{mg}$, showed significant increases in median LBM compared with baseline: $1.5 \mathrm{~kg}$ (95\% CI, $2.1-12.6 \mathrm{~kg} ; P=0.0012)$ and $1.0 \mathrm{~kg}(95 \% \mathrm{CI},-4.8-11.5$ $\mathrm{kg} ; P=0.046)$ respectively, with no improvement observed in the placebo arm. (median increase, $0.02 \mathrm{~kg}$ [95\% CI, -5.8-6.7], $P=0.88)$. Median time to climb 12 stairs also significantly decreased in enobosarm $1 \mathrm{mg}(P$ $=0.0019)$ and $3 \mathrm{mg}(P=0.0065)$ but not in the placebo arm $(P=0.26)$. QOL assessed by Functional Assessment of Anorexia/Cachexia Therapy (FAACT) score also improved significantly in the enobosarm $1 \mathrm{mg}$ and $3 \mathrm{mg}$ arms. No comparison was presented between the two groups of enobosarm [38].

\section{Combination treatments}

Several studies used combination of agents to treat cancer cachexia.

\section{$\mathrm{MA}+$ thalidomide}

In an RCT, 102 patients with advanced cancer of any type and cachexia ( $\geq 5 \%$ loss of body weight) were randomized to receive MA $160 \mathrm{mg}$ orally twice per day or MA $160 \mathrm{mg}$ orally twice per day plus thalidomide $50 \mathrm{mg}$ orally twice per day for 8 weeks. Primary endpoints were body weight, fatigue as measured by Multidimensional Fatigue Symptom Inventory-Short Form (MFSI-SF) scale, and QOL assessed by EORTC QOL-C30 form. Both groups showed improvement in weight and fatigue before and after treatment. The mean change from baseline in the body weight $(P=0.05)$, fatigue $(P<0.01)$, QOL $(P=0.01)$, HGS $(P=0.05)$ and ECOG performance status in the combination group were significantly greater than in MA-alone group; however, a direct comparison between the groups was not presented [41]. Similarly significant improvements in levels of IL-6 and TNF-a was observed in the MA-Thalidomide group as compared to MA group.

\section{$\mathrm{MA}+$ meloxicam + eicosapentaenoic acid (EPA)}

In an RCT, 62 patients with advanced cancer and cancer cachexia ( $\geq 5 \%$ loss of body weight) were randomized to receive 1) MA + non-steroidal anti-inflammatory (meloxicam), 2) $\mathrm{MA}+$ meloxicam + oral EPA, or 3) meloxicam + EPA for a treatment duration of 3 months. The primary efficacy endpoints of body weight and LBM improved in all three arms compared to baseline, with no significant difference among the three treatment groups $(P=0.61)$ [42]. 


\section{$\mathrm{MA}+\mathrm{EPA}+\mathrm{L}$-carnitine + thalidomide}

In this trial, 332 patients with advanced cancer and cancer cachexia ( $\geq 5 \%$ loss of body weight) were randomized to receive 1) medroxyprogesterone $(500 \mathrm{mg} /$ day) or MA (320 mg/day), 2) EPA, 3) L-carnitine, 4) thalidomide 200 $\mathrm{mg} /$ day, or 5) a combination of the above for 4 months. The primary endpoints were increase in LBM, a decreased in resting energy expenditure (REE), and a decrease in fatigue. At two interim analyses after 125 and 204 patients, arms 1 and 2 were withdrawn due to significant inferiority for primary endpoints compared to the other arms. A post hoc analysis among arms 3, 4, and 5 showed significant improvement in LBM, REE), appetite and fatigue $(P=0.035)$ in arm 5 as compared to arms 3 and 4. There was no difference in HGS among the groups $(P=0.399)$ [43]. Similarly, significant improvements in IL-6 and TNF-a levels were observed in arms 5. No significant differences in overall survival were observed between arms 3,4 , and 5 .

\section{L-carnitine + celecoxib + MA}

In a phase III randomized non-inferiority trial, 60 patients with advanced cancer and cancer cachexia $(\geq 5 \%$ loss of body weight) were randomized to receive either 1) L-carnitine $4 \mathrm{~g} /$ day + celecoxib $300 \mathrm{mg} /$ day or 2) L-carnitine $4 \mathrm{~g} /$ day + celecoxib $300 \mathrm{mg} /$ day $+\mathrm{MA}$ $320 \mathrm{mg} /$ day. All patients received nutritional supplements as well. The primary endpoint was improvement in LBM and total daily physical activity. There was no significant difference between the groups in LBM, physical activity, QOL, handgrip strength or overall survival [44].

\section{Celecoxib+MA}

In a phase III randomized trial, 96 patients diagnosed with gastrointestinal malignancies ( $>5 \%$ of unintentional weight loss in the past 6 months) were randomized to either combination of 1) celecoxib (200 mg/day) and MA $(320 \mathrm{mg} / \mathrm{d})$ or 2) MA $(320 \mathrm{mg} / \mathrm{d})$ plus placebo for 8 weeks [45]. The primary endpoint was change in body weight. Secondary outcomes included changes in quality of life, grip strength, appetite score, performance status, plasma albumin, CRP, IL-6, and Glasgow Prognostic Score. Though improvements were seen in weight change across both groups, no significant differences were observed between groups. Similarly, no significant differences were observed between two groups on secondary outcomes [45].

\section{Quality assessment}

We applied GRADE criteria for measuring risk of bias in our studies. We applied categories used to measure bias in GRADE to our studies, and measured risk of bias based on these criteria (Table 2). Of 19 included studies,
5 studies reported high degree of evidence, 9 studies reported moderate degree of evidence and 5 were rated as providing low degree of evidence.

\section{Discussion}

The management of cancer cachexia remains an unmet need in the field of oncology. Coupled with disease burden, management of cachexia poses significant challenge due to underlying multisystem pathways that play role in inducing cachexia. In addition, differences in outcomes based on type and stage of disease poses additional challenge. Our systematic review identified 19 studies (representing 20 RCTs) that focused on pharmacological agents in clinical trials to manage cancer cachexia. Anamorelin, as a single agent showed promising results and was associated with significant improvement in body weight in all 5 studies. In addition, enobosarm $1 \mathrm{mg}$ and $3 \mathrm{mg}$ showed significant improvements in weight as compared to baseline, however no group comparisons were available. Finally, combination therapy with MA and thalidomide showed improvements in cachexia associated symptoms, though these were not significant. A multidrug approach using combination of progestins (MA or MPA) plus EPA plus thalidomide and L-carnitine was also associated with significant improvement in body weight and other symptoms as compared to individual arms, highlighting the need to target multiple pathways underlying cachexia among cancer patients. In addition, only insulin was associated with improvement in overall survival among cancer patients with cachexia. None of the agents showed improvement in progression-free survival. Finally we also observed that the clinical definition of cachexia also varied between studies Our results provide an update to the previous systematic review of 2005 that identified progestins such as MA and short-course corticosteroids as beneficial in managing this condition, and identified newer agents including anamorelin, as future promising agents for managing cachexia among cancer patients [19].

A symposium conducted by the University of Rochester Cancer Center Community Oncology Program on Cancer Cachexia and Sarcopenia identified key areas for future research in area of cancer cachexia. These include: incorporating morphometrics into clinical decision making, focusing on identifying and treating patients at precachexia stages, and identifying long term biomarkers that serve as markers of changes from precachexia to cachexia, expand patient selection in cachexia trials, and identifying and incorporating realistic endpoints into clinical trials including patient reported outcomes [4]. An important development in the research into cancer cachexia was the acceptance of uniform criteria for its clinical diagnosis by Fearon et al in 2011 [5]. Now a uniform selection criterion can be applied for clinical trials instead of investigator-dependent criteria, 
Table 2 Risk of Bias: Evidence assessment using the GRADE Criteria

\begin{tabular}{|c|c|c|c|c|c|c|c|c|c|c|}
\hline $\begin{array}{l}\text { Author } \\
\text { (Year) }\end{array}$ & \begin{tabular}{|l} 
Lack of \\
allocation \\
concealmen \\
$t$
\end{tabular} & $\begin{array}{l}\text { Lack } \\
\text { of } \\
\text { Blindi } \\
\text { ng }\end{array}$ & $\begin{array}{l}\text { Incomplete } \\
\text { accounting of patients } \\
\text { and outcome events }\end{array}$ & $\begin{array}{l}\text { Selective outcome } \\
\text { reporting (AE or } \\
\text { Survival Not reported) }\end{array}$ & $\begin{array}{l}\text { Other } \\
\text { limitati } \\
\text { ons }\end{array}$ & \begin{tabular}{|l|} 
External \\
Validity/Generaliz \\
ability/Applicability
\end{tabular} & \begin{tabular}{|l|} 
Differenc \\
es in \\
populatio \\
$\mathrm{n}$
\end{tabular} & $\begin{array}{l}\text { Imprecision: } \\
\text { Small } \\
\text { sample size }\end{array}$ & $\begin{array}{l}\text { Publication } \\
\text { Bias: Selective } \\
\text { Reporting }\end{array}$ & $\begin{array}{l}\text { Final Judgement Level } \\
\text { of Evidence)-High, } \\
\text { Moderate, Low, }\end{array}$ \\
\hline $\begin{array}{l}\text { Del } \\
\text { Fabbro } \\
\text { (2013) }\end{array}$ & No & No & No & Yes & Yes & No & No & No & Yes & Low \\
\hline $\begin{array}{l}\text { Dobs } \\
\text { (2013) }\end{array}$ & Maybe & No & No & No & No & Maybe & Yes & No & No & Low \\
\hline $\begin{array}{l}\text { Garcia } \\
\text { (2015) }\end{array}$ & No & No & No & Yes & No & Maybe & Yes & No & Yes & Moderate \\
\hline $\begin{array}{l}\text { Gordon } \\
\text { (2005) }\end{array}$ & No & No & No & No & No & No & No & No & No & High \\
\hline $\begin{array}{l}\text { Hong- } \\
\text { Sheng } \\
(2013)\end{array}$ & No & No & No & Yes & No & Maybe & Yes & No & Yes & Moderate \\
\hline $\begin{array}{l}\text { Jatoi } \\
\text { (2007) }\end{array}$ & No & No & No & No & No & Maybe & Yes & No & No & Moderate \\
\hline $\begin{array}{l}\text { Jatoi } \\
\text { (2010) }\end{array}$ & No & No & No & No & No & No & No & No & No & High \\
\hline $\begin{array}{l}\text { Kanat } \\
(2013)\end{array}$ & No & No & No & Yes & No & Maybe & Yes & No & Yes & Moderate \\
\hline $\begin{array}{l}\text { Katakami } \\
\text { (2018) }\end{array}$ & No & No & No & No & No & No & No & No & No & Low \\
\hline $\begin{array}{l}\text { Kouchaki } \\
(2018)\end{array}$ & No & No & No & No & No & No & No & Yes & No & High \\
\hline $\begin{array}{l}\text { Lundholm } \\
\text { (2007) }\end{array}$ & No & No & No & Yes & No & No & No & No & Yes & Moderate \\
\hline $\begin{array}{l}\text { Madeddu } \\
\text { (2012) }\end{array}$ & No & No & No & No & No & Maybe & Yes & No & No & Moderate \\
\hline $\begin{array}{l}\text { Mantovan } \\
\text { i (2010) }\end{array}$ & No & No & No & Yes & No & Maybe & Yes & No & Yes & Moderate \\
\hline $\begin{array}{l}\text { Mehrzad } \\
(2016)\end{array}$ & No & No & No & Yes & No & Maybe & Yes & No & Yes & Low \\
\hline $\begin{array}{l}\text { Strasser } \\
(2006)\end{array}$ & No & No & No & Yes & No & Maybe & Yes & No & Yes & Moderate \\
\hline $\begin{array}{l}\text { Touos) } \\
\text { Takayam } \\
\text { a (2016) }\end{array}$ & No & No & No & No & No & No & No & No & No & High \\
\hline $\begin{array}{l}\text { Temel } \\
(2016)\end{array}$ & No & No & No & No & No & No & No & No & No & High \\
\hline $\begin{array}{l}\text { Turcott } \\
(2018)\end{array}$ & No & No & Maybe & Yes & Yes & No & Yes & Yes & Yes & Low \\
\hline $\begin{array}{l}\text { Yelisuraj } \\
\text { alingam } \\
\text { (2012) }\end{array}$ & No & No & No & Yes & No & Maybe & Yes & Yes & Yes & Moderate \\
\hline
\end{tabular}

Green: No Risk of Bias; Yellow: Maybe Risk of Bias; Red: Risk of Bias Present. In column on final judgement: Red: Low level of Evidence: Yellow: Moderate level of evidence: Green: High Level of Evidence

which varied between the trials. Because our review extends back to 2004, many studies adopted a variable definition of cancer cachexia, which makes comparisons between patient populations and pharmacological agents difficult. Moreover, the definition proposed by Freon et al. remains very simplistic as it does not capture the full spectrum of cancer cachexia and fails to differentiate the degree of cancer cachexia severity. Another challenge in cancer cachexia clinical trials is the need to demonstrate improvements in body weight, muscle mass, and functional outcome with simultaneous utilization of systemic chemotherapeutic agents, as many of these agents are associated with concomitant weight loss among cancer patients, possibly masking the effect of cancer cachexia agents. More comprehensive guidelines are needed to incorporate additional biomarkers or measurements of cachexia including body composition and muscle mass into future clinical practice and clinical trials in management of cancer cachexia [46].

In our review, use of ghrelin agonist anamorelin as a single agent showed significant benefit in improvement in LBM. In all 5 included studies, anamorelin (in doses of 50 and $100 \mathrm{mg}$ ) for 12 weeks was associated with significant improvement in weight and appetite compared with placebo [25-27]. Anamorelin is an orally active, high-affinity, selective ghrelin-receptor agonist [27]. Ghrelin is normally secreted by gastric endocrine cells and acts as a ligand for growth hormone, thereby increasing IGF1-concentrations, which has both direct and indirect effects on muscle growth and production of anti-cachectic cytokines [25]. In mouse models, ghrelin was shown to increase appetite and food intake and simultaneously down-regulate production of IL- 6 , IL- $1 \alpha$, $-1 \beta$, and TNF- $\alpha$ [47]. These cytokines have been implicated in the development of cachexia, as they are responsible for reducing weight, inhibiting appetite, and increasing resting energy expenditure [25]. Due to the short half-life of parental ghrelin $(30 \mathrm{~min})$ and no benefit from its use in the management of cachexia, the use of oral synthetic ghrelin is effective. In addition, the correlation of anamorelin with increased IGF-1 and IGFBP3 levels further supports the anabolic effects of anamorelin. Anamorelin, however, failed to show improvement in function as measured by HGS. Perhaps a better approach is to measure functional improvement is by utilizing a pedometer to document improvement in number of steps taken every day by the patients.

Multiple pathways underlying cachexia have been identified through murine models, including signaling pathways via tumor necrosis factor receptor superfamily 
member 12A, role of parathyroid hormone in influencing metabolic states or energy wasting through white adipose tissue through inactivation of $5^{\prime}$ adenosine monophosphate-activated protein kinase [48]. Inflammation too is a key driver in cancer cachexia possibly through increased expression of pro-inflammatory cytokines like IL-6 and TNF-a and induction of acute phase protein response, a key marker of systemic inflammation [49]. TNF- $\alpha$ is a cytokine implicated in systemic inflammation and cancer cachexia. Etanercept is a dimeric fusion protein consisting of the extracellular ligand-binding portion of the human $75-\mathrm{kD}$ TNF receptor linked to the Fc portion of human immunoglobulin IgG1 [32]. Hence, several agents such as etanercept, infliximab, thalidomide, and pentoxifylline were investigated as potential agents to manage cachexia and impact inflammatory pathways. However, none could demonstrate any significant improvement in patient weight or associated symptoms. In addition, thalidomide did not show any improvement in levels of proinflammatory cytokines [35].Other agents, including those targeting the IL-6 pathway, such as tocolizumab, are good candidates for future trials [50].

Corticosteroids and anabolic androgenic steroids have been shown to have mixed effects on increasing body fat mass among cancer patients. However, due to an absence of tissue specificity and increased side effects, their use can be limited [51]. Hence, selective androgen receptors like enobosarm have been recently developed. Enobosarm (GTx-024; GTx, Memphis, TN, USA) is a selective androgen receptor modulator that induces conformational changes in the androgen receptor upon binding. Subsequently, it selectively alters the interaction of the receptor with coactivator and corepressor proteins that exist in different tissues and changes the receptor's ability to regulate gene expression [38]. Enobosarm (1 and $3 \mathrm{mg}$ ) resulted in improved LBM and improved physical function in patients with cancer with existing cachexia (muscle loss), with tolerable side effects compared to placebo. However, in preliminary reports from 2 subsequent phase III clinical trials (POWER1 and POWER2), Enobosarm (3 mg) failed to show an improvement in bodyweight and chair rise function, however showed significant improvement in lean body mass [52]. The results of POWER1 and POWER2 are not yet published.

Since cachexia is a multi-organ syndrome affecting multiple metabolic pathways, it may be prudent to do combination studies involving more than one agent (appetite stimulant + anti-inflammatory + anabolic agent) to demonstrate improvements in weight gain as well as functional improvement. Five studies were identified that involved multiple agents for management of cachexia. Use of nutrition supplements with celecoxib and megesterol acetate provided no benefits in either cachexia related symptoms or reducing levels of inflammatory cytokines. On the other hand, treatment with megesterol acetate and thalidomide showed borderline improvements in cachexia associated symptoms including fatigue and inflammatory markers among patients with advanced cancer, with no significant difference on weight change [41]. Megesterol acetate is a steroidal progestin derivative of progesterone, and used as appetite stimulant among cancer patients [47]. Thalidomide shows multiple anti-inflammatory and immunomodulatory roles, affects levels of inflammation by reducing production of IL- 6 and TNF-a, and shows positive results in management of cachexia [41]. Finally, multidrug treatment with thalidomide, EPA, progestins and L-carnitine showed promising results in multiarmed trial on cachexia management among patients with advanced cancer. Perhaps combining more efficacious agents like thalidomide or anamorelin with Enobosarm or tocilizumab may result not only in improvement of weight but also clinically meaningful function. None of the agents studied in these clinical trials demonstrated significant toxicity, which also makes a case for conducting combination studies.

Our study has several strengths. We focused our search on pharmacological management and only included clinical trials to have a defined approach. We restricted our data abstraction to original peer reviewed literature, and all previous conference proceedings and reviews were excluded. Finally, we did not restrict our search to any cancer type or stage, to get an adequate representation of cachexia among cancer patients. Our study has several limitations. We focused primarily on phase III or advanced clinical trials and might have missed out on preliminary studies that can prove effective agents for cachexia. Second, we did not include any studies that focused on nutrition or exercise interventions, except for those that were used as additional agents in combination agent RCTs, and hence further studies are needed to understand the impact of these nutrition/exercise-based interventions on cachexia associated outcomes. Finally, studies using thalidomide faced challenges including low sample size and short term follow up, hence their results need validation in future multicenter clinical trials.

Cancer cachexia remains a challenging condition and poses significant burden on health among patients diagnosed with advanced cancer. It not only impacts their overall QOL but can impact their overall survival and response to chemotherapeutic agents. Though we could not identify or can recommend a standard treatment of cachexia, treatment with agents like Anamorelin and Enobosarm show promising results. Anamorelin, a ghrelin agonist has the best evidence for efficacy in cancer cachexia, but further research is needed to 
demonstrate its clinically meaningful benefit. In addition, multidrug therapy can impact underlying mechanisms of cachexia and improve QOL among cancer patients. Future multicenter trials examining various combinations of agents with anamorelin might pave wave for developing an effective gold standard for managing cachexia in cancer patients. Further, solely relying on BMI as an endpoint of cachexia should be limited, as owing to the growing epidemic of obesity, the proportion of patients presenting with severe weight loss and BMI $<20$ continue to decline, and hence incorporating changes in muscle mass is crucial to study comprehensive effect of pharmacological agents on cachexia management [4]. Additionally, more biomarker driven clinical trials are needed in field of cancer cachexia to prospectively track changes in biomarkers associated with changes in body composition or inflammatory markers among cancer patients. Our findings support the need of innovative strategies including current clinical trials under development comprising of multimodal therapy like the MENAC trial comprising of combination of exercise, nutrition and anti-inflammatory medication to target multiple underlying mechanism including reducing inflammation, improve anabolism and promote energy and protein balance [53]. Further, clinical trials in pre-cachexia phases using these identified agents may help provide long term options for management of cachexia, address symptoms at an earlier stage for overall improvement in QOL and clinical outcomes.

\section{Conclusion}

Our review identified anamorelin as a single agent showing promising results for managing cachexia. Further, more evidence and studies are needed to demonstrate long term efficacy of agents like enobosarm and combination agents like MA and thalidomide. Further, combining with exercise/ \& or nutrition intervention may provide additional benefit.

\section{Additional files}

Additional file 1: Summary of Databases Searched. (DOCX 33 kb)

Additional file 2: Impact on pharmacological agents on cachexia associated biomarkers. (DOCX $19 \mathrm{~kb}$ )

\footnotetext{
Abbreviations

ASAS: Anderson Symptom Assessment Scale; BAS: Best available support; CE: Cannabis extract; ESAS: Edmonton Symptom Assessment Scale; FAACT: Quality of life by the Functional Assessment of Anorexia/Cachexia Therapy (FAACT) questionnaire; LBM: Lean body mass; MA: Megesterol acetate; MPA: Medroxyprogesterone acetate; N/A: Not available; NS: Not significant; NSCLC: Non-small cell lung cancer; PL: Placebo; QOL: Quality of life; QOL-ACD: Quality of Life-Anti Cancer Drugs; RCT: Randomized controlled trial; THC: Tetrahydrocannabinol; VAS: Visual analog scale
}

\section{Funding}

No funding to declare.

Availability of data and materials

We have cited all previously published studies included in our study.

\section{Authors' contributions}

SA: Planning, design, acquisition of data, analysis and manuscript writing; PA: Planning, design, analysis and manuscript writing; HV: Acquisition of data and manuscript writing; SJ: Design, acquisition of data, analysis and manuscript writing. All authors read and approved the final manuscript.

Ethics approval and consent to participate

No approval was required now as the study was focused on conducting a systematic review of previously published literature.

\section{Consent for publication}

Not applicable.

\section{Competing interests}

The authors declare that they have no competing interests.

\section{Publisher's Note}

Springer Nature remains neutral with regard to jurisdictional claims in published maps and institutional affiliations.

\section{Author details}

${ }^{1}$ Division of Oncology, Lombardi Comprehensive Cancer Center, Georgetown, University School of Medicine, Washington DC 20007, USA. ${ }^{2}$ Radiation Epidemiology Branch, Division of Cancer Epidemiology and Genetics, National Institutes of Health, National Cancer Institute, Rockville, MD 20850, USA. ${ }^{3}$ The University of Texas Health Science Center School of Public Health, Houston, TX 77030, USA. ${ }^{4}$ Department of Medicine, Division of Oncology, The University of Texas Health Science Center at Houston, McGovern Medical School, Houston, TX 77030, USA.

Received: 27 March 2018 Accepted: 12 November 2018 Published online: 27 November 2018

\section{References}

1. Stephens NA, Skipworth RJ, Fearon KC. Cachexia, survival and the acute phase response. Curr Opin Support Palliat Care. 2008;2:267-74.

2. Zilbermint MF, Dobs AS. Nonsteroidal selective androgen receptor modulator Ostarine ${ }^{\mathrm{TM}}$ in cancer cachexia. Future Oncol. 2009;5:1211-20.

3. Mantovani G. Randomised phase III clinical trial of 5 different arms of treatment on 332 patients with cancer cachexia. Eur Rev Med Pharmacol Sci. 2010;14:292-301.

4. Dunne RF, Mustian KM, Garcia JM, et al. Research priorities in cancer cachexia: the University of Rochester Cancer Center NCl Community Oncology Research Program Research Base Symposium on Cancer Cachexia and sarcopenia. Curr Opin Support Palliat Care. 2017;11:278-86.

5. Fearon K, Strasser F, Anker SD, et al. Definition and classification of cancer cachexia: an international consensus. Lancet Oncol. 2011;12:489-95.

6. Madeddu C, Mantovani G. An update on promising agents for the treatment of cancer cachexia. Curr Opin Support Palliat Care. 2009;3:258-62

7. Madeddu C, Maccio A, Panzone F, et al. Medroxyprogesterone acetate in the management of cancer cachexia. Expert Opin Pharmacother. 2009;10: 1359-66.

8. Maltoni M, Nanni O, Scarpi E, Rossi D, Serra P, Amadori D. High-dose progestins for the treatment of cancer anorexia-cachexia syndrome: a systematic review of randomised clinical trials. Annals of Oncology. 2001; 12(3):289-300

9. Giordano KF, Jatoi A. The cancer anorexia/weight loss syndrome: therapeutic challenges. Curr Oncol Rep. 2005;7:271-6.

10. Mateen F, Jatoi A. Megestrol acetate for the palliation of anorexia in advanced, incurable cancer patients. Clin Nutr. 2006:25:711-5.

11. Laviano A, Meguid MM, Inui A, et al. Therapy insight: Cancer anorexiacachexia syndrome--when all you can eat is yourself. Nat Rev Clin Oncol. 2005;2:158.

12. Dewys WD, Begg C, Lavin PT, et al. Prognostic effect of weight loss prior tochemotherapy in cancer patients. Am J Med. 1980;69:491-7. 
13. Gullett NP, Hebbar G, Ziegler TR. Update on clinical trials of growth factors and anabolic steroids in cachexia and wasting. Am J Clin Nutr. 2010;91: 1143S-7S.

14. Morley JE, Thomas DR, Wilson M-MG. Cachexia: pathophysiology and clinical relevance. Am J Clin Nutr. 2006;83:735-43.

15. Taylor $L A$, Pletschen $L$, Arends J, et al. Marine phospholipids_-a promising new dietary approach to tumor-associated weight loss. Support Care Cancer. 2010;18:159

16. Doyle A, Zhang G, Fattah EAA, et al. Toll-like receptor 4 mediates lipopolysaccharide-induced muscle catabolism via coordinate activation of ubiquitin-proteasome and autophagy-lysosome pathways. FASEB J. 2011;25: 99-110.

17. Zhang G, Jin B, Li YP. C/EBP $\beta$ mediates tumour-induced ubiquitin ligase atrogin1/MAFbx upregulation and muscle wasting. EMBO J. 2011;30:4323-35.

18. Zhang G, Liu Z, Ding H, et al. Toll-like receptor 4 mediates Lewis lung carcinoma-induced muscle wasting via coordinate activation of protein degradation pathways. Sci Rep. 2017;7:2273.

19. Yavuzsen T, Davis MP, Walsh D, et al. Systematic review of the treatment of cancer-associated anorexia and weight loss. J Clin Oncol. 2005;23:8500-11.

20. VonVille H. Excel workbooks for systematic reviews; 2015.

21. Balshem $H$, Helfand $M$, Schünemann $H J$, et al. GRADE guidelines: 3. Rating the quality of evidence. J Clin Epidemiol. 2011;64:401-6.

22. Strasser F, Luftner D, Possinger $K$, et al. Comparison of orally administered cannabis extract and delta-9-tetrahydrocannabinol in treating patients with cancer-related anorexia-cachexia syndrome: a multicenter, phase III, randomized, double-blind, placebo-controlled clinical trial from the Cannabis-in-Cachexia-study-group. J Clin Oncol. 2006;24:3394-400.

23. Turcott JG, Núñez MRG, Flores-Estrada D, et al. The effect of nabilone on appetite, nutritional status, and quality of life in lung cancer patients: a randomized, double-blind clinical trial. Support Care Cancer. 2018;26:3029. https://doi.org/10.1007/s00520-018-4154-9.

24. Del Fabbro E, Dev R, Hui D, et al. Effects of melatonin on appetite and other symptoms in patients with advanced cancer and cachexia: a double-blind placebo-controlled trial. J Clin Oncol. 2013:31:1271-6

25. Garcia JM, Boccia RV, Graham CD, et al. Anamorelin for patients with cancer cachexia: an integrated analysis of two phase 2, randomised, placebocontrolled, double-blind trials. Lancet Oncol. 2015;16:108-16.

26. Takayama K, Katakami N, Yokoyama T, et al. Anamorelin (ONO-7643) in Japanese patients with non-small cell lung cancer and cachexia: results of a randomized phase 2 trial. Support Care Cancer. 2016:24:3495-505.

27. Temel JS, Abernethy AP, Currow DC, et al. Anamorelin in patients with nonsmall-cell lung cancer and cachexia (ROMANA 1 and ROMANA 2): results from two randomised, double-blind, phase 3 trials. Lancet Oncol. 2016;17:519-31.

28. Currow D, Temel J, Abernethy A, et al. ROMANA 3: a phase 3 safety extension study of anamorelin in advanced non-small-cell lung cancer (NSCLC) patients with cachexia. Ann Oncol. 2017;28:1949-56.

29. Katakami N, Uchino J, Yokoyama T, et al. Anamorelin (ONO-7643) for the treatment of patients with non-small cell lung cancer and cachexia: results from a randomized, double-blind, placebo-controlled, multicenter study of Japanese patients (ONO-7643-04). Cancer. 2018;124:606-16

30. Bai Y, Hu Y, Zhao Y, et al. Anamorelin for cancer anorexia-cachexia syndrome: a systematic review and meta-analysis. Support Care Cancer. 2017:25:1651-9.

31. Nishie K, Yamamoto S, Nagata C, et al. Anamorelin for advanced non-smallcell lung cancer with cachexia: systematic review and meta-analysis. Lung Cancer. 2017;112:25-34.

32. Jatoi A, Dakhil SR, Nguyen PL, et al. A placebo-controlled double blind trial of etanercept for the cancer anorexia/weight loss syndrome. Cancer. 2007; 110:1396-403.

33. Jatoi A, Ritter $H L$, Dueck A, et al. A placebo-controlled, double-blind trial of infliximab for cancer-associated weight loss in elderly and/or poor performance non-small cell lung cancer patients (N01C9). Lung Cancer. 2010;68:234-9.

34. Gordon J, Trebble T, Ellis $\mathrm{R}$, et al. Thalidomide in the treatment of cancer cachexia: a randomised placebo controlled trial. Gut. 2005:54:540-5.

35. Yennurajalingam S, Willey JS, Palmer $\mathrm{JL}$, et al. The role of thalidomide and placebo for the treatment of cancer-related anorexia-cachexia symptoms: results of a double-blind placebo-controlled randomized study. J Palliat Med. 2012:15:1059-64.

36. Mehrzad V, Afshar R, Akbari M. Pentoxifylline treatment in patients with cancer cachexia: a double-blind, randomized, placebo-controlled clinical trial. Adv Biomed Res. 2016;5:60.
37. Lundholm K, Körner U, Gunnebo L, et al. Insulin treatment in cance cachexia: effects on survival, metabolism, and physical functioning. Clin Cancer Res. 2007;13:2699-706.

38. Dobs AS, Boccia RV, Croot CC, et al. Effects of enobosarm on muscle wasting and physical function in patients with cancer: a double-blind, randomised controlled phase 2 trial. Lancet Oncol. 2013;14:335-45.

39. Dalton JT, Barnette KG, Bohl CE, et al. The selective androgen receptor modulator GTx-024 (enobosarm) improves lean body mass and physical function in healthy elderly men and postmenopausal women: results of a double-blind, placebo-controlled phase II trial. J Cachexia Sarcopenia Muscle. 2011:2:153-61.

40. Bohl CE, Chang C, Mohler ML, et al. A ligand-based approach to identify quantitative structure- activity relationships for the androgen receptor. J Med Chem. 2004;47:3765-76.

41. Wen H-S, Li X, Cao Y-Z, et al. Clinical studies on the treatment of cancer cachexia with megestrol acetate plus thalidomide. Chemotherapy. 2012;58: 461-7.

42. Kanat $\mathrm{O}$, Cubukcu E, Avci $\mathrm{N}$, et al. Comparison of three different treatment modalities in the management of cancer cachexia. Tumori. 2013:99:229-33.

43. Mantovani G, Macciò A, Madeddu C, et al. Randomized phase III clinical trial of five different arms of treatment in 332 patients with cancer cachexia. Oncologist. 2010;15:200-11.

44. Madeddu C, Dessi M, Panzone F, et al. Randomized phase III clinical trial of a combined treatment with carnitine+ celecoxib \pm megestrol acetate for patients with cancer-related anorexia/cachexia syndrome. Clin Nutr. 2012;31: $176-82$.

45. Kouchaki B, Janbabai G, Alipour A, et al. Randomized double-blind clinical trial of combined treatment with megestrol acetate plus celecoxib versus megestrol acetate alone in cachexia-anorexia syndrome induced by Gl cancers. Support Care Cancer. 2018;26:2479-89.

46. Roeland EJ, Ma JD, Nelson SH, et al. Weight loss versus muscle loss: reevaluating inclusion criteria for future cancer cachexia interventional trials. Support Care Cancer. 2017:25:365-9.

47. Guillory B, Splenser A, Garcia J. The role of ghrelin in anorexia-cachexia syndromes. In: Vitamins \& Hormones: Elsevier; 2013;92:61-106. Academic Press.

48. Graf SA, Garcia JM. Anamorelin hydrochloride in the treatment of cancer anorexia-cachexia syndrome: design, development, and potential place in therapy. Drug Des Devel Ther. 2017;11:2325-31.

49. Deans C, Wigmore SJ. Systemic inflammation, cachexia and prognosis in patients with cancer. Curr Opin Clin Nutr Metab Care. 2005:8:265-9.

50. Ando K, Takahashi F, Motojima S, et al. Possible role for tocilizumab, an anti-interleukin-6 receptor antibody, in treating cancer cachexia. J Clin Oncol. 2012:31:e69-72

51. Bhasin S, Calof OM, Storer TW, et al. Drug insight: testosterone and selective androgen receptor modulators as anabolic therapies for chronic illness and aging. Nat Rev Endocrinol. 2006;2:146.

52. Crawford J, Johnston M, Hancock M, et al. Enobosarm, a selective androgen receptor modulator (SARM) increases lean body mass (LBM) in advanced NSCLC patients: updated results of two pivotal, international phase 3 trials. Support Care Cancer. 2014;22:S30

53. Solheim TS, Laird BJ, Balstad TR, et al. Cancer cachexia: rationale for the MENAC (Multimodal_Exercise, Nutrition and Anti-inflammatory medication for Cachexia) trial. BMJ Support Palliat Care. 2018. https://doi.org/10.1136/ bmjspcare-2017-001440.

Ready to submit your research? Choose BMC and benefit from:

- fast, convenient online submission

- thorough peer review by experienced researchers in your field

- rapid publication on acceptance

- support for research data, including large and complex data types

- gold Open Access which fosters wider collaboration and increased citations

- maximum visibility for your research: over $100 \mathrm{M}$ website views per year

At BMC, research is always in progress.

Learn more biomedcentral.com/submission 